Background Children's breast preference, feeding from one breast more than the other, has been thought to be common. It may develop spontaneously in absence of known risk factors. We know of no study that explores characteristics of spontaneous children's breast preference (BP).

Objective To establish characteristics of spontaneous BP among children aged 2-24 months.

Design/methods We conducted this cross-sectional study through self-administered survey in 8 primary healthcare centres in AlAhsa area, Saudi Arabia. A convenience sample of 500 mothers who brought their 2-24 months old children for vaccination was recruited during 3 months of 2013. The survey consisted of two parts: part 1 was general demographic questions and part 2 explored more about BP. The survey was validated by 2 lactation consultants and tested in a pilot sample of 20 mothers. Surveys with nonresponses on presence or absence of BP question were excluded whereas other nonresponses were replaced by multiple imputation.

Results A total of 480 mothers answered the question on presence or absence of BP (response rate 96\%). Out of this, 127 (26\%) mothers reported that their children had BP. Prevalence of spontaneous BP was 14\% (67/480). Self-reported causes of BP differed significantly (Chi-Square goodness-of-fit $\mathrm{p}<0.001$ ): spontaneous BP constituted $53 \%$ of the causes; small nipple $19 \%$; less milk production 17\%; various medical/surgical pathologies 6\%; mothers' preference 5\%. Mothers observed that spontaneous BP began at a median of 1 month (interquartile range: 1 day-3 months, maximum: 9 months). No significant sex predominance was noted (binomial $\mathrm{p}=0.50$ ). Proportion of children preferred right or left breast were similar (42\% vs 58\% binomial $\mathrm{p}=0.50)$. Spontaneous BP was the main or ancillary reason for $21 \%$ of formula milk usage within the first six months of life. Nineteen mothers were worried from spontaneous BP; however, only 7 of them sought medical advice.

Conclusions Spontaneous BP was the most common cause of BP in children aged 2-24 months with prevalence of $14 \%$ and with no sex or side predominance. It started at very early in life and negatively impacted exclusive breastfeeding. Further study is warranted to confirm these results in other populations and ascertain biological causes and the best intervention, as this could help to improve exclusive breastfeeding.

\section{P0-0101 PROGRESSIVE FAMILIAL INTRAHEPATIC CHOLESTASIS IN SAUDI ARABIA}

A Almehaidib. Pediatrics, King Faisal Specialist Hospital and Research Center, Riyadh, Saudi Arabia

\subsection{6/archdischild-2014-307384.770}

Background Progressive familial intrahepatic cholestasis (PFIC) is a rare inherited autosomal recessive disease where patients develop severe cholestasis progressing to biliary cirrhosis and chronic liver failure, usually during the first decade of life.

Aim To review the clinical presentation, familial involvement and outcome of patients with PFIC in Saudi Arabia.

Methods A retrospective study for patients who were diagnosed to be PFIC based on gene study between 2000-2013.

Results 48 patients were confirmed by gene test to have PFIC: 5/type 1, 27/type 2, and 16/type 3 .

Consanguinity is seen in all patients (100\%) and 31 patients (64\%) having positive family history of liver diseases All PFIC type 1 patients presented with jaundice, poor growth, hepatomegaly, normal hearing and GGT. Diarrhoea in 4 patients $(80 \%)$
Two patients (40\%) underwent liver transplantation. Of the 27 patients with PFIC type 2, 24 patients (88\%) with jaundice 22 patients $(81 \%)$ and itching 3 patients (11\%), hepatomegaly in 23 patients $(85 \%)$, and 6 patients $(22 \%)$ with signs of rickets. Biochemically three patients (11\%) have high GGT on presentation and the rest of patients (88\%) had normal GGT and normal cholesterol and 9 patients $(33 \%)$ had coagulopathy (INR $>1.3$ ). Fifteen patients $(55 \%)$ underwent liver transplantation; three patients (11\%) died.

16 patients with PFIC type 3 presented after 2 years of age, 6 patients (37\%) with only jaundice in 5 patients (31\%) and 4 patients $(25 \%)$ with only itching, hepatomegaly in 14 patients (93\%) and one patient (6\%) with signs of rickets. Biochemically one patient (6\%) had normal GGT, and two patients (12\%) had coagulopathy on presentation. One patient $(6 \%)$ underwent liver transplantation.

Conclusion The vast majority of patients with PFIC type 1 and 2 present in the first two years and have normal GGT, while type 3 present after two years and have high GGT. However, GGT can be high in PFIC type $2(11 \%)$ as it can be normal in PFIC type 3 (6\%). One third of patients required liver transplant.

\section{PO-0102 TRANSCUTANOUS INTRAHEPATIC PORTOSYSTEMIC SHUNT (TIPSS) IN CHILDREN}

A Almehaidib. Pediatrics, King Faisal Specialist Hospital and Research Center, Riyadh, Saudi Arabia

\subsection{6/archdischild-2014-307384.771}

Background Variceal haemorrhage is a major cause of mortality and morbidity in patients with portal hypertension. Transcutaneous intrahepatic portosystemic shunt (TIPSS) is a procedure which is widely used in adults with limited experience in children.

Objectives The aim of this study is to analyse the use of preand post-operative laboratory results as predictors for TIPSS failure in the given sample.

Methods This is a retrospective study of children who underwent TIPSS from 1998 to 2013. Data were obtained within 1 month before TIPSS placement. The variables are shown in (Table 1). Failure of TIPSS was defined as requirement of revision within 36 months after the procedure. Patients were divided into two groups post-operatively according to the procedure success; those who did not require revision within 36 months after procedure were considered to have successful TIPSS, while those who needed revision within 36 months after procedure had a failed TIPSS procedure.

Abstract P0-0102 Table 1 Characteristics of the study sample and success rate

\begin{tabular}{|l|c|}
\hline Age in month & $n=10(\%)$ \\
\hline $78-166$ & 112.4 \\
-Cause of Liver disease & $($ mean) \\
Congenital Hepatic Fibrosis & $5(50)$ \\
Allagile Syndrome & $1(10)$ \\
Autto-immune hepatitis & $1(10)$ \\
Wilson disease & $1(10)$ \\
Budd-Chiani Syndrome & $1(10)$ \\
Cryptogenic liver cirrthosis & $1(10)$ \\
-Indication for TIPSS & \\
Variceal Bleeding & $10(100)$ \\
-Out-come at 36 months after procedure & \\
Success, no revision after 36 month & $7(70)$ \\
Revision in 36 months & $3(30)$ \\
Recurrent Bleeding & $2(20)$ \\
Occlusion & $1(1)$ \\
\hline
\end{tabular}


Abstract P0-0102 Table 2 p values of pre- and post-operative variables in association with TIPSS failure

\begin{tabular}{|c|c|c|c|c|c|c|c|c|}
\hline \multirow{2}{*}{} & \multicolumn{3}{|c|}{ Successful TIPSS } & \multicolumn{3}{c|}{ Failed/Revised TIPSS } \\
\cline { 2 - 9 } & Pre-Operative & \multicolumn{2}{|c|}{ Post-operative } & \multicolumn{2}{|c|}{ Pre-operative } & \multicolumn{2}{c|}{ Post-operative } \\
\cline { 2 - 9 } & Mean & $\boldsymbol{P}$ value & Mean & $\boldsymbol{P}$ value & Mean & P value & Mean & $\boldsymbol{P}$ value \\
\hline Haemoglobin & 10.33 & 0.43 & 11.6 & 0.137 & 11.33 & 0.526 & 13.3 & 0.319 \\
\hline Platelets & 95.67 & 0.48 & 113 & 0.42 & 92.17 & 0.325 & 94.3 & 0.546 \\
\hline WBC & 9.33 & 0.318 & 4.62 & 0.474 & 5.83 & 0.158 & 4.6 & 0.614 \\
\hline ALT & 81.33 & 0.588 & 43.9 & 0.563 & 71.83 & 0.58 & 52.33 & 0.592 \\
\hline AST & 46 & 0.599 & 58.5 & 0.611 & 46.3 & 0.653 & 35.6 & 0.447 \\
\hline ALP & 145.33 & 0.105 & 275.5 & 0.615 & 372.17 & $0.027 *$ & 182.3 & 0.521 \\
\hline GGT & 22 & 0.426 & 56.7 & 0.316 & 36.5 & 0.451 & 105 & 0.354 \\
\hline PT & 12 & 0.505 & 16.9 & 0.582 & 15 & 0.392 & 18.7 & 0.681 \\
\hline PIT & 37.67 & 0.776 & 39.7 & 0.055 & 37.5 & 0.871 & 53.6 & 0.268 \\
\hline INR & 1.33 & 0.12 & 1.3 & 0.133 & 1.5 & 0.25 & 1.5 & 0.423 \\
\hline Urea & 3.33 & 0.656 & 3.9 & 0.634 & 3.33 & 0.653 & 4.38 & 0.607 \\
\hline Creatinine & 26 & 0.485 & 45.8 & 0.46 & 36.17 & 0.466 & 53.3 & 0.623 \\
\hline
\end{tabular}

Results A total of 10 children (7 females) underwent TIPSS. The primary indication was variceal haemorrhage in all the patients with different diagnoses (Table-1). Congenital Hepatic Fibrosis was the most common aetiology (50\%). Three patients $(30 \%)$ needed revision in a mean of 24.2 month post-procedure. 7 patients $(70 \%)$ did not require revision 36 month post procedure, with a mean follow up of 60.5 month.

Pre-operative high serum alkaline phosphatase (ALP) was associated with TIPSS failure ( $\mathrm{p}$ value $=0.027$ ) and thus, can be used as a pre-operative predictor for failed TIPSS. Other variables did not show significant association with TIPSS procedure including patients age and weight.

Conclusion This study involves the association of multiple variables with TIPSS outcomes in children. ALP showed a relative association as a predictor to TIPSS failure. Further studies are needed with a larger sample.

\section{PO-0103 EVALUATION OF THE PAEDIATRIC PATIENTS WITH GASTROINTESTINAL BLEEDING: EXPERIENCE OF A TERTIARY CENTRE}

T Ozkan, D Altay, A Otuzbir, T Ozgur. Pediatric Gastroenterology Hepatology and Nutrition, Uludag University Medical Faculty, Bursa, Turkey

\subsection{6/archdischild-2014-307384.772}

Introduction Gastrointestinal bleeding (GB) can be seen in children of all ages and it is one of the frequent application reason to paediatric gastroenterologists. Even though the causes of bleeding differs according to age groups, it may become lifethreatening depending on the severity of the bleeding.

Aim The aim of the study was to determine the demographical and etiological factors of patients who admitted to our clinic with upper or lower gastrointestinal bleeding.

Material and method 94 patients, were included to the study, admitted to Uludag University Faculty of Medicine Paediatric Gastroenterology department with upper or lower gastrointestinal bleeding between January 2010 and June 2013. Patients' files were evaluated retrospectively.

Results The number of patients with upper gastrointestinal bleeding (UGB) was 53, average ages of these patients was 11.1 years (2-18 years), $45.3 \%$ were female and $54.7 \%$ were male, respectively. At the aetiology of these bleeding cases H.pylori were detected in $18.8 \%$, peptic or duodenal ulcer were detected in 10 patients (range of ages 10-18 years, average 12 years) and H.pylori was detected in only two patients with ulcer. While all gastric mucosa were hyperemic in 26 patients, distal esophagitis or duodenitis were detected also in 23 patients with UGB.
Esophageal variceal bleeding was the cause of 4 patients with UGB and chronic renal failure was associated to bleeding in one patient. Barrett's oesophagus was detected histologically in 10 and 13 years of age two patients. Four patients had a history of nonsteroidal anti-inflammatory drug use prior to bleeding. Henoch-Schonlein disease was diagnosed in 7 years old male patient. Bleeding was the first symptom of this disease in this case and purpura was occurred after 4 days of bleeding. The remaining 41 cases were called idiopathic bleeding. Range of the ages of 41 patients with lower gastrointestinal bleeding (LGB) was 11.1 years and 24 patients were male $(58.5 \%$ male, $41.5 \%$ female, respectively). Ulcerative colitis was diagnosed histologically in 9 patients (21.9\%). Polip in rectum or sigmoid colon was detected in 6 patients, nonspesific chronic inflammation was reported pathologically in patients with polip and there were no family history for polip in these patients. Colonoscopy was normal in 13 patients (31.7\%) with LGB.

Conclusion Chronic gastritis was detected majority in aetiology of the patients with UGB and we thought that bad dietary habits had great importance in these cases. $21.9 \%$ cases with LGB were diagnosed with ulcerative colitis. All cases were evaluated, there were no death because of bleeding at the prognosis of these patients.

Discussion Gastrointestinal bleeding is one of important reason for reference of tertiary centre in children. Considering the frequency of H.pylori positivity in our society, $18.8 \%$ were found to be positive in cases with UGB. Although the vast majority of patients with LGB were idiopatic, ulcerative colitis plays an important role in patients with LGB.

\section{PO-0104 DIFFERENT CLINICAL SPECTRUM OF CYTOMEGALOVIRUS HEPATITIS IN INFANTS}

T Ozkan, D Altay, A Otuzbir, U Sahin, T Ozgur. Pediatric Gastroenterology Hepatology and Nutrition, Uludag University Medical Faculty, Bursa, Turkey

\subsection{6/archdischild-2014-307384.773}

Background and aim Cytomegalovirus (CMV) is, a member of the herpes viridae family, found widely in nature and the most common congenital infection in newborns. The average incidence of CMV infection in newborn infants is $1 \%$. Irreversible signs of central nervous system involvement (microcephaly, deafness, mental-motor retardation) develops in 5-10\% congenitally infected infants. Signs of perinatal infections, hepatosplenomegaly, pneumonia, hepatitis, are seen, but in this period neurological sequelae are rare. In this study, CMV-infected patients who were admitted to our clinic within 3 months were examined.

Methods Between the date of December 2013 and February 2014, in total five CMV infection ( $\min 45$ days, max 2 years-4 months old) have been detected at Uludag University, Faculty of Medicine, Department of Paediatric Gastroenterology. Three of those patients admitted with jaundice and other two patients were detected during the pancytopenia aetiology and vomiting aetiology investigation.

In our series, which consist of developed CMV hepatitis cases due to different etiological reasons, retrospective examination is conducted with clinical and laboratory findings.

Results Biliary atresia was detected in three patients. One is by intraoperative cholangiography and other two, as evidenced by histopathology. In the fourth case, CMV infection was detected simultaneously with vitamin $\mathrm{B}_{12}$ deficiency during pancytopenia investigation. In the fifth patient, performed liver transplantation due to neonatal hepatitis, CMV infection was detected during 